\title{
47. The Sheaf of Relative Canonical Forms of a Kähler Fiber Space over a Curve
}

\author{
By Takao FuJITA \\ Department of Mathematics, College of General Education, \\ University of Tokyo, Komaba, Meguro, Tokyo 153 \\ (Communicated by Kunihiko KodaIRA, M. J. A., Sept. 12, 1978)
}

In this note we announce an improvement of a result in [1]. Details shall be published elsewhere.

A triple $f: M \rightarrow S$ of a holomorphic mapping $f$ and compact complex manifolds $M, S$ is called a Kähler fiber space if $M$ is Kähler, $f$ is surjective and any general fiber of $f$ is connected. By $\omega_{M / S}$ we denote the relative dualizing sheaf $\omega_{M} \otimes f^{*} \omega_{S}^{\smile}=\mathcal{O}_{M}\left[K_{M}-f^{*} K_{S}\right]$. Then we have the following

Theorem. Let $f: M \rightarrow C$ be a Kähler fiber space over a curve $C$. Then $f_{*} \omega_{M / C} \cong \mathcal{O}_{C}[A \oplus U]$ for an ample vector bundle $A$ and a flat vector bundle $U$ on $C$.

For a proof, we show the following lemma and use the criterion of Hartshorne [4].

Lemma. Let $E$ be the vector bundle such that $f_{*} \omega_{M / C} \cong \mathcal{O}_{C}[E]$. Then $\operatorname{deg}(\operatorname{det} Q) \geqq 0$ for any quotient bundle $Q$ of $E$. Moreover, if $\operatorname{deg}(\operatorname{det} Q)=0$, then $Q$ is a direct sum component of $E$ and has a flat connection.

Outline of the proof of lemma. Let $S$ be the image of singular fibers of $f$ and let $C^{o}=C-S$. Note that the restriction $E_{C^{\circ}}$ of $E$ to $C^{o}$ is isomorphic to the bundle $\bigcup_{x \in C_{0}} H^{n, 0}\left(F_{x}\right)$, where $F_{x}=f^{-1}(x)$ and $n=\operatorname{dim} F_{x}$. Hence $E_{C^{o}}$ has a natural Hermitian structure. This defines a Hermitian structure of $Q_{C o}$ in a canonical manner. Let $\Omega$ be the Chern De Rham curvature form representing $c_{1}\left(Q_{C^{\circ}}\right)$. Then we have the following formula: $\operatorname{deg}(\operatorname{det} Q)=\int_{C o} \Omega+\sum_{p \in S} e_{p}$, where $e_{p}$ is the local exponent of $\operatorname{det} Q$ at $p \in S$ (see [3]). Similarly as in [1], we prove that $\Omega$ is semi-positive and that $e_{p} \geqq 0$ for any $p \in S$. So $\operatorname{deg}(\operatorname{det} Q)$ $\geqq 0$. Moreover, if $\operatorname{deg}(\operatorname{det} Q)=0$, then $\Omega \equiv 0$ and $e_{p}=0$ for any $p$. $\Omega \equiv 0$ implies that the orthogonal complements $\tilde{Q}_{x}\left(x \in C^{\circ}\right)$ of $\operatorname{Ker}\left(E_{x}\right.$ $\left.\rightarrow Q_{x}\right)$, considered as subspaces of $H^{n, 0}\left(\boldsymbol{F}_{x}\right) \subset H^{n}\left(\boldsymbol{F}_{x} ; \boldsymbol{C}\right)$, form a flat subbundle of the flat bundle $\bigcup_{x \in C^{o}} H^{n}\left(F_{x} ; C\right)$. So, $Q_{C^{\circ}}$ is isomorphic to the vector bundle $\tilde{Q}_{o}=\bigcup_{x \in C^{\circ}} \tilde{Q}_{x}$ associated with the monodromy action of $\pi_{1}\left(C^{o}, x_{o}\right)$ on $\tilde{Q}_{x_{o}} \subset H^{n}\left(F_{x_{o}} ; C\right)$, where $x_{o}$ is a point on $C^{o}$. Now, $e_{p}=0$ 
implies that the local monodromy of $\tilde{Q}_{x_{o}}$ at $p$ is trivial and that the splitting $Q_{C^{\circ}} \cong \tilde{Q}_{C^{\circ}} \subset E_{C^{\circ}}$ can be extended over $p$. Thus we prove the lemma.

As an application, we can prove the following

Theorem. Let $f: M \rightarrow C$ be an algebraic fiber space over a curve. Suppose that the canonical bundle of any general fiber of $f$ is trivial. Then $\kappa\left(M, \omega_{M / C}\right) \geqq 0$. Moreover, the equality holds if and only if $f$ is birationally equivalent to a holomorphic fiber bundle over $C$.

\section{References}

[1] T. Fujita: Kähler fiber spaces over curves (to appear in J. Math. Soc. Japan).

[2] _ : On the theory of Kodaira dimension (in Japanese) (to appear in Sugaku).

[3] — : On local exponents (in preparation).

[4] R. Hartshorne: Ample vector bundles on curves. Nagoya Math. J., 43, $73-89$ (1971). 